\title{
THE GHOST OF AN INDEX THEOREM
}

\author{
ROBIN HARTE
}

(Communicated by Paul S. Muhly)

\begin{abstract}
The "index theorem" whose spirit we invoke says that the index of a product of Fredholm operators is the sum of their indexes; our extension is to operators with "generalized inverses".
\end{abstract}

0 . Call the bounded linear operator $T: X \rightarrow Y$ between normed spaces regular if there is another bounded linear operator $T^{\prime}: Y \rightarrow X$ for which

$$
T=T T^{\prime} T
$$

then $T^{\prime}$ is called a generalized inverse, or pseudo-inverse, for $T$ ([1], [3], [4], [5], [7]). It is familiar that we can always arrange

$$
T^{\prime}=T^{\prime} T T^{\prime}:
$$

indeed if (0.1) holds with $T^{\prime}=T^{\prime \prime}$ then (0.1) and (0.2) hold with $T^{\prime}=T^{\prime \prime} T T^{\prime \prime}$.

1. Lemma. If $T=T T^{\prime} T: X \rightarrow Y$ is regular, with $T^{\prime}=T^{\prime} T T^{\prime}$, then,

$$
T(X) \cong T^{\prime}(Y) \text {. }
$$

Proof. The mappings $T^{\wedge}: T^{\prime}(Y) \rightarrow T(X)$ and $T^{\prime \wedge}: T(X) \rightarrow T^{\prime}(Y)$ induced by $T$ and $T^{\prime}$ are mutually inverse.

If $T=T T^{\prime} T: X \rightarrow Y$ and $S=S S^{\prime} S: Y \rightarrow Z$ are both regular then the product $S T: X \rightarrow Z$ may or may not be regular ([1, $\S 5.1]$; [3, Example 4.6]); necessary and sufficient is that the product $S^{\prime} S T T^{\prime}: Y \rightarrow Y$ is regular ([1, Chapter 5 Theorem 1]; [3, Theorem 1.4]; [4, Theorem 3.8.3]). Thus if (1.2)

$$
T=T T^{\prime} T: X \rightarrow Y, \quad S=S S^{\prime} S: Y \rightarrow Z \quad \text { and } \quad S T=S T U S T: X \rightarrow Z
$$

are all regular, we may assume that we have arranged

$$
T^{\prime}=T^{\prime} T T^{\prime}, \quad S^{\prime}=S^{\prime} S S^{\prime}, \quad U=U S T U \text { and } U=T^{\prime} V S^{\prime} .
$$

With these preparations, we are ready to meet our ghost:

Received by the editors November 8, 1988 .

1980 Mathematics Subject Classification (1985 Revision). Primary 47B30, 46B30; Secondary 47A10, 47A53. 
2. Theorem. If $T: X \rightarrow Y, S: Y \rightarrow Z$ and $S T: X \rightarrow Z$ are regular then there is isomorphism

$$
T^{-1}(0) \times S^{-1}(0) \times Z / S T(X) \cong(S T)^{-1}(0) \times Y / T(X) \times Z /(S(Y)) .
$$

Proof. If generalized inverses $T^{\prime}, S^{\prime}$ and $U$ for $T, S$ and $S T$ satisfy the conditions (1.3), look at the mappings

$$
\mathbf{U}=\left(\begin{array}{ccc}
I-T^{\prime} T & 0 & 0 \\
(I-T U S) T & I-T U S & 0 \\
0 & S(I-T U S) & I-S S^{\prime}
\end{array}\right):\left(\begin{array}{c}
X \\
Y \\
Z
\end{array}\right) \rightarrow\left(\begin{array}{c}
X \\
Y \\
Z
\end{array}\right)
$$

and

$$
\mathbf{V}=\left(\begin{array}{ccc}
I-T^{\prime} T & T^{\prime}\left(I-S^{\prime} S\right) & 0 \\
0 & \left(I-T T^{\prime}\right)\left(I-S^{\prime} S\right) & \left(I-T T^{\prime}\right) S^{\prime} \\
0 & 0 & I-S S^{\prime}
\end{array}\right):\left(\begin{array}{c}
X \\
Y \\
Z
\end{array}\right) \rightarrow\left(\begin{array}{c}
X \\
Y \\
Z
\end{array}\right)
$$

We have

$$
\begin{aligned}
\mathbf{V U} & =\left(\begin{array}{ccc}
I-U S T & 0 & 0 \\
0 & I-T T^{\prime} & 0 \\
0 & 0 & I-S S^{\prime}
\end{array}\right) \\
\text { and } \quad \mathbf{U V} & =\left(\begin{array}{ccc}
I-T^{\prime} T & 0 & 0 \\
0 & I-S^{\prime} S & 0 \\
0 & 0 & I-S T U
\end{array}\right),
\end{aligned}
$$

and hence also

$$
\mathbf{U}=\mathbf{U V U} \text { and } \mathbf{V}=\mathbf{V U V}
$$

Applying Lemma 1 with $\mathbf{U}$ and $\mathbf{V}$ in place of $T$ and $T^{\prime}$, and inspecting the ranges of $\mathbf{U}$ and $\mathbf{V}$, gives (2.1).

If $T$ and $S$ and hence also $S T$ are Fredholm then the subspaces in (2.1) are all finite dimensional, and hence the isomorphism (2.1) says that both sides are of equal dimension. To say that the difference between the dimensions on each side of (2.1) is zero is to state the "index theorem" for Fredholm operators in its usual form. Of course the essence of Theorem 2 holds in a general additive category [3], in particular for the elements of a ring.

The operators which go to make up the matrices $\mathbf{U}$ and $\mathbf{V}$ come from the exact sequence used in the "one-diagram" proof of the index theorem due to Yang [8]; the process of converting a split-exact sequence and its "inverse" into mutually invertible matrices comes from Putinar ([6, (2.3) pages $72-73])$. It is tempting to express the four subspaces of $Y$ used in the more usual proof of the index theorem ([2, Theorem 3.2.1]; [4, Theorem 6.5.4]; [5, Theorem 23.1]; [7, Theorem IV.13.1]) as the ranges of projections derived from $T^{\prime}, S^{\prime}$ and $U:$ for example

$$
S^{-1}(0) \cap T(X)=(I-T U S) T T^{\prime}(Y)
$$


and

$$
Y=\left(S^{-1}(0)+T(X)\right) \oplus S^{\prime} S(I-T U S)(Y) .
$$

The projections $U S T, T U S$ and $S T U$ also perform the splitting of $X, Y$ and $Z$ used in the proof of Satason [9].

\section{REFERENCES}

1. S. R. Caradus, Operator theory of the pseudo-inverse, Queen's Papers in Pure and Appl. Math. no. 50, Queen's University, Kingston, Ontario 1978.

2. S. R. Caradus, W. E. Pfaffenberger and B. Yood, Calkin algebras and algebras of operators on Banach spaces, Dekker, New York, 1974.

3. R. E. Harte, Fredholm, Weyl and Browder theory, Proc. Roy. Irish Acad. Sect. A, 85 (1986), 151-176.

4. __ Invertibility and singularity for bounded linear operators, Dekker, New York, 1988.

5. H. G. Heuser, Functional analysis, Wiley, New York, 1982.

6. M. Putinar, Some invariants for semi-Fredholm systems of essentially commuting operators, Jour. Op. Theory 8 (1982), 65-90.

7. A. E. Taylor and D. C. Lay, Introduction to functional analysis, Wiley New York, 1980.

8. K.-W. Yang, Index of Fredholm operators, Proc. Amer. Math. Soc. 41 (1973), 329-330.

9. D. Sarason, The multiplication theorem for Fredholm operators, Amer. Math. Monthly 94 (1987), 68-70.

Department of Mathematics, University College of Cork, Cork, Ireland 